\title{
Applications of Spatial Object Modelling in Fuzzy Topological Space for Land Cover Changes
}

\author{
H.C. Chamuah \\ Research Scholar \\ Dibrugarh University, Dibrugarh \\ Assam, India
}

\author{
B.C. Chetia \\ Principal, \\ North Lakhimpur College (Autonomous) \\ Lakhimpur, Assam, India
}

\begin{abstract}
There have been many research developments on the conceptual description of topological relation between spatial objects. Fuzzy spatial objects have become more and more important in GIS application when spatial phenomena are generalised by the crisp form a large of quantitative information is lost. Land Cover as a fuzzy spatial, temporal object should be modelled in a fuzzy framework. The paper is an attempt to construct topological relation as one of the most important characteristic of spatial -temp oral object with its application to land cover. Here we consider on area of Dhemaji District which has different land cover type to construct topological relationship between them both spatially and temporally.
\end{abstract}

\section{Keywords}

Fuzzy topology, fuzzy spatial objects GIS Land cover, image classification.

\section{INTRODUCTION}

GIS application plays extremely important role in fuzzy spatial object modelling. Fuzzy spatial objects uncertainly should be considered in all aspect of a GIS to have a better understanding of the real world. Fuzzy spatial objects those with intermediate boundary, hence the objects have some degree of membership belonging to a category.

In order to define and model fuzzy spatial object such as land covers, it is necessary to investigate their fuzzy topological relations Topology is one of the internal concept of geographical information system. Generally speaking, it deals with the structural representation of spatial features and than their properties that remain invariant under certain transformation. Topology and topological relationship have a wide range of applications in robotics, artificial intelligence and GIS. Topological relationship are non metric properties of geospatial objects that remain constant when the object space is continuously distorted (Tang 2004). Methods of constructing topological relationship can be categorize into two classes name point based methods such as 9 intersection matrices that can be based on crisp set or a fuzzy set theory. 4 intersection and 9 intersection approaches in a connected topological space by using the interiors boundaries and exteriors between two crisp subsets was introduced by Egenhofer and Franzosa (1991) and Egenhofer and Herring (1990). Eight relations have been identified between two simple regions in the two dimensional Euclidean space. In addition, thirteen topological relations between two temporal internals were identified by Allen using temporal operators. Begin and end which provide respectively the beginning and ending instances of a time interval.

These approaches do not consider the uncertainty and fuzziness in any aspect of a spatio-temporal object. In order to deal with the fuzziness of the spatial objects, Tang (2004) defined boundary of the boundary region and interior of the boundary. Based on these core concepts $5 * 5$ intersection matrixes are defined. Malek (2004) has modelled spatial relationships using intuitionistic fuzzy topology.

A new method is suggested to construct topological relations for land covers as spatio-temporal objects land use and land cover; most of which is obtained from the classification results of satellite images; many be good example of a fuzzy spatial objects. As a case study a past of Dhemaji District which has different parcels and also different land cover types is selected Fundamental concepts for uncertainty modelling of spatial relationships are analyzed from the view point of fuzzy logic. It is demonstrated that how fuzzy logic can provide a model for fuzzy region; i.e. regions with indeterminate boundaries.

General, after classification, such pixel in the image is assigned to particulars land cover type; therefore a pixel belongs to one and only one type (Tang 2004). In reality all pixels contains a member of different contributing land use types. In general, land cover is continuously distributed in nature and there is seldom a clear boundary between different natural phenomena. In this study land cover as a spatial fuzzy objects is modelled. Results are scientifically assessed in the paper and showed the superiority of the model over conventional ones. 


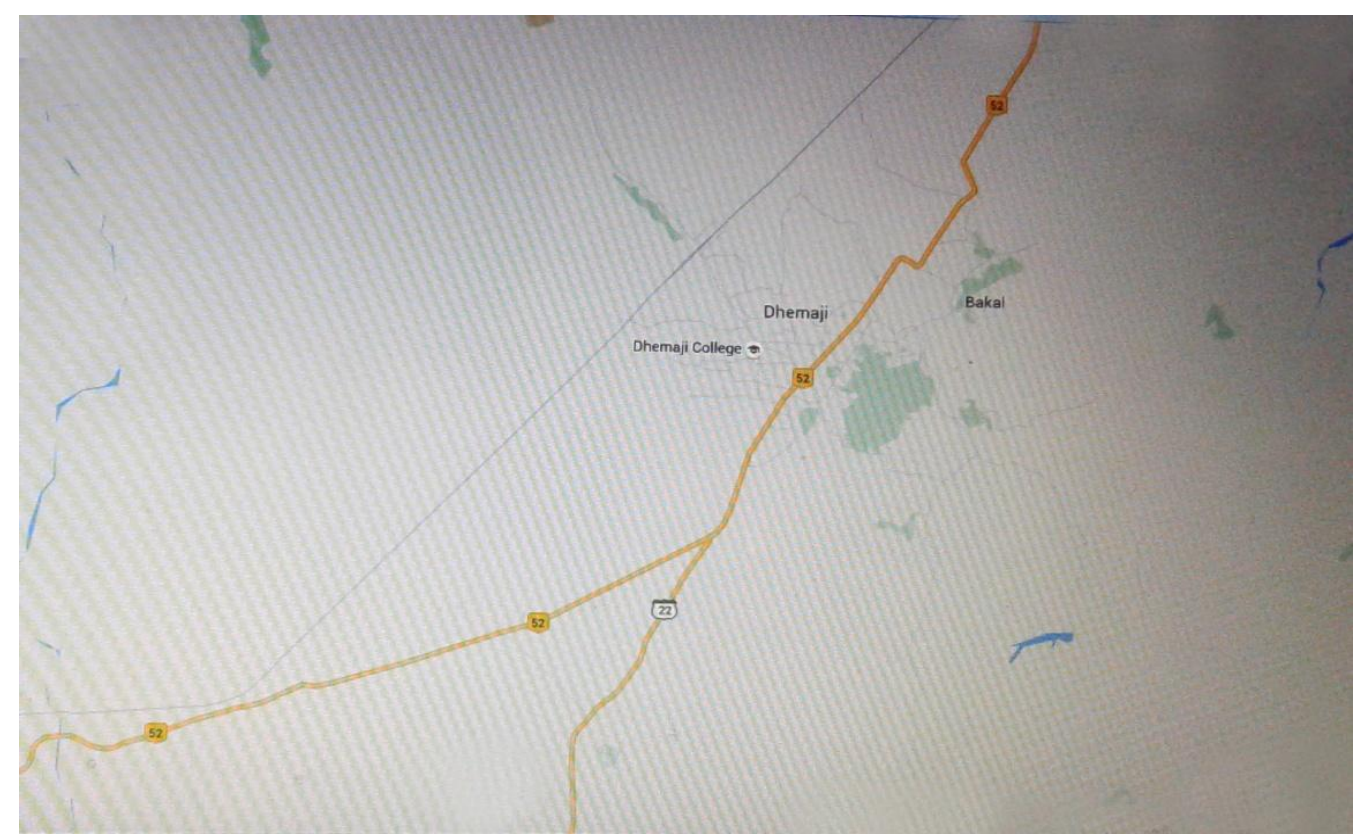

Figure 1: Study area Dhemaji District

\section{STUDY SITE}

Dhemaji district is an administrative district in the eastern part of slate of Assam in India. The total land area accepted by the district is $3237 \mathrm{~km} 2$ with population of 688,077 (as 2011). The district headquarters are located at Dhemaji. It is one of the most remote districts of India, at the easternmost part of Assam. Situated in the foothills of the lower Himalayas it is relatively a small town.

\section{FUZZINESS}

The information that possessed about the real world is almost uncertain incomplete and imprecise Fuzziness may include five aspects; inaccuracy and error vagueness, incompleteness, inconsistency and imprecision (Worboys 1998)

In order to deal with fuzziness, Zadeh proposed the famous fuzzy set theory (Zadeh 1985). The fuzzy set and fuzzy logic are the most powerful tools for solving these fuzzy problems.

Zadeh generalized a fuzzy set from classical set theory by allowing intermediate situations between the whole and nothing. For a fuzzy set, a membership function is defined to describe the degree of an element to a class. The membership value ranges from 0 to 1 where 0 shows that the element does not belong to a class, 1 means "belongs" and other values indicate the degree of membership to a class.

Fuzzy set theory is the extension of classical set theory by allowing the membership of elements to range from 0 to 1.Let $\mathrm{x}$ be the universe of a classical set of objects. Membership in a classical subset A of $\mathrm{X}$ is often viewed as a characteristic function $\mu(\mathrm{x})(\mathrm{x}$ is a generic element of $\mathrm{X})$ from $X$ to $\{0,1\}$ (Dubois and Prade 1980) $\{0,1\}$ is called a valuation set. If the valuation set is allowed to be the real interval $[0,1], \mathrm{A}$ is called a fuzzy set. $\mu(\mathrm{x})$ is the membership value (or degree of membership) of $\mathrm{x}$ in $\mathrm{A}$. Clearly, $\mathrm{A}$ is a subset of $\mathrm{X}$ that has no sharp boundary. A fuzzy set A can be represented by the set of pairs:

$$
\mathrm{A}=\{(x, \mu(x), x \in X\}
$$

\section{FUZZY TOPOLOGY Fuzzy sets and fuzzy topology}

A fuzzy set in $\mathrm{X}$ is a function with domain $\mathrm{X}$ and value in $\mathrm{I}$, that is an element of $I^{X}$. Let $\mathrm{A} \in \mathrm{IX}$. The subset of $\mathrm{X}$ in which A assumes non-zero values in known as the support of A. For every $\mathrm{x} \in \mathrm{X}, \mathrm{A}(\mathrm{x})$ is called the grade of membership of $\mathrm{x}$ in $\mathrm{A}$. $\mathrm{X}$ is called the carrier of the fuzzy set $\mathrm{A}$. If $\mathrm{A}$ takes only the values 0 and 1 , then $\mathrm{A}$ is called a crisp set in $\mathrm{X}$.

Let $A, B \in I^{\mathrm{X}}$ we define the following fuzzy sets.

1. $\mathrm{A}=\mathrm{B}$ if $\mu_{A}(\mathrm{x})=\mu_{B}(\mathrm{x})$ for all $\mathrm{x}$ in $\mathrm{X}$.

2. $\mathrm{A} \leq \mathrm{B}$ if $\mu_{A}(\mathrm{x}) \leq \mu_{B}(\mathrm{x})$ for all $\mathrm{x}$ in $\mathrm{X}$.

3. $\mathrm{C}=\mathrm{AV}$ Bif $\mu_{C}(\mathrm{x})=\max \left\{\mu_{A}(\mathrm{x}), \mu_{B}(\mathrm{x})\right.$ for all $\mathrm{x}$ in $\left.\mathrm{X}\right\}$

4. $\mathrm{A} \wedge \mathrm{B} \in \mathrm{I}^{\mathrm{x}}$ by $(\mathrm{A} \wedge \mathrm{B})(\mathrm{x})=\min \{\mathrm{A}(\mathrm{x}), \mathrm{B}(\mathrm{x})$, for every $\mathrm{x} \in \mathrm{X}$ (intersection) $\}$.

Fuzzy sets, open fuzzy sets and closed fuzzy sets are the basic elements of fuzzy topology. In the following, we introduce the concept of fuzzy topology.

\subsection{Fuzzy Topological Space}

Let $\mathrm{X}$ be a non empty ordinary set and $\mathrm{I}=[0,1], \partial \subset I^{X}, \partial$ is called a I-fuzzy topology on $\mathrm{X}$, and $\left(I^{X}, \partial\right)$, is called Ifuzzy topological space (I-fts), if $\partial$ satisfies the following conditions:

$$
\begin{array}{cl}
\text { i. } & 0,1 \in \partial ; \\
\text { ii. } & \text { If } \mathrm{A}, \mathrm{B} \in \delta \text {, then } \mathrm{A} \wedge \mathrm{B} \in \partial . \\
\text { iii. } & \text { Let }\left\{A_{i}: i \in \mathrm{J}\right\} \subset \partial \text {, where } \mathrm{J} \text { is an index set, } \\
\text { then } \vee_{i \in J} A_{i} \in \partial
\end{array}
$$

Where $0 \in \partial$ means the empty set and $1 \in \partial$ means the whole set $\mathrm{X}$. 
The elements in $\partial$ are called open elements and the elements in the component of $\partial$ are called closed elements and the set of the complement of open set is denoted by $\partial^{\prime}{ }^{\prime} \cdot$.

\subsection{Definition}

For any fuzzy set A, we define.

i. The interior of $\mathrm{A}$ the joining of all the open subsets contained in $\mathrm{A}$, denoted by $A^{0}$, i.e $A^{0}$. $=\mathrm{V}\{\mathrm{B} \in \partial ; \mathrm{B} \leq \mathrm{A}\}$

ii. The closure of $\mathrm{A}$ as the meeting of all the closed subsets containing $\mathrm{A}$, denoted by $\bar{A}$, i.e. $\overline{\mathrm{A}}=\Lambda\left\{\mathrm{B} \in \partial^{\prime}: \mathrm{B} \geq \mathrm{A}\right\}$

iii. The exterior of $\mathrm{A}$ is the complement of the closure of A.

Figure is given below

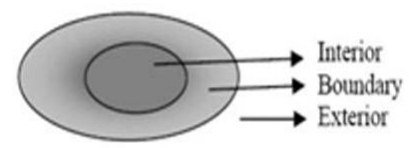

The figure 2, indicates the concept of interior, exterior and boundary of a set.

\subsection{Definition}

For any fuzzy set A. We define the complements of A by $\mathrm{A}^{\prime}(\mathrm{x})=1-\mathrm{A}(\mathrm{x})$

\subsection{Definition}

The boundary of a fuzzy se $\mathrm{A}$ is defined $\partial \mathrm{A}=\overline{\mathrm{A}} \Lambda A^{-c}$

The above are the commonly used definition of fuzzy topological space, fuzzy interior, fuzzy closure, fuzzy complement and fuzzy boundary (Liu and Luo, 1997).

The boundary of a subset may also have its interior and its boundary of the boundary. On the other hand, the interior and the closure of a subset also have their boundaries. For example the boundary of the boundary of a fuzzy set is the union of the boundary of the closure and the boundary of the interior of a fuzzy set. i e $\partial(\partial A)=\partial\left(A^{-}\right) \cup \partial\left(A^{0}\right)$.

From the above information one can define a maximum of 5 areas for each objects: interior, boundary of the boundary, interior of the boundary, boundary of the interior and rest are exterior.

Consequently one can develop the traditional 9 intersection matrix into $5 * 5$ intersection ones.

\section{GENERATING FUZZY LAND COVER OBJECT AND THEIR TOPOLOGICAL RELATIONSHIP.}

The importance of land cover needs no more explanation. Since, it plays a fundamental role in lots of fields. Land resources pose one of the biggest problems all around the world.

The method for forming fuzzy land cover for spot image is addressed.

First of all these image were classified. Because the membership value was needed, so some special programmes were developed to achieve the purpose. The pixel into the land cover classes can be assigned and have their membership. It seems to have asset whose elements are the pair of pixel and their membership. So the basis of the study is the fuzzy set. It can be defined the interior and the boundary of the boundary, and boundary of the interior and the exterior base on the pixels membership.

The pixels whose memberships are between 1 and 0.8 are categorized into interior of each area interval. The pixels are between 0.8 and 0.6 are categorized into boundary of the interior of each interval. The pixel whose memberships are between 0.4 and 0.6 are categorised interior of the boundary of each interval. The boundary of the boundary region includes the pixels with membership value between 0.2 to 0.4 and the next are categorised into exterior of each interval. So it is possible to show the relationship between the regions into the $5 * 5$ intersection matrix.

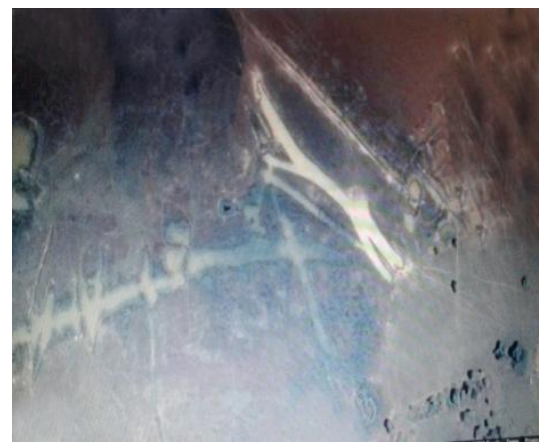

Figure 3: Image of Study area

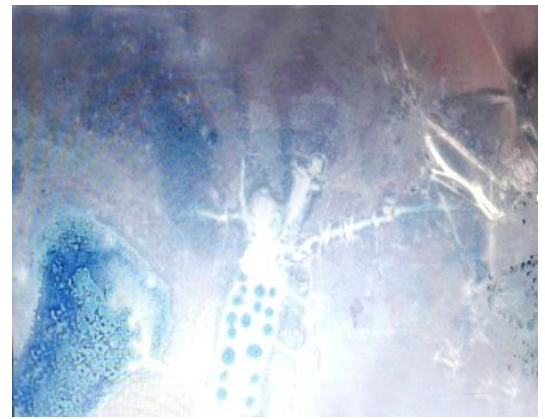

Figure 4: Image classification result

Each region is valid for an interval. The land cover map is the basis for dynamic analysis of land use and land covers. The methods usually compare the different base on a crisp pixel by - pixel method. But in this way the judgment is more precise. The update image is possible to find the valid time interval of the regions. Based on Allen's temporal operators, End and Begin temporal Topological relation can be deduced.

The figure 4, shows that five interval were categorised. For each interval interior, interior of the boundary, boundary of the interior, boundary of boundary and rest interval exterior are determined.

\section{CONCLUSION}

Fuzzy spatial object of Land cover is a model in a fuzzy framework. Further the relationships between these fuzzy spatial object are much more complicated than between crisp spatial object. This paper assign in particular area the pixel into the landcover classes and have their fuzzy 
membership value. The pixel whose memberships are categorized into five classes for constructing topological relation between region which is essential. In this way five area can be considered and 5*5 intersection matrix in found.

\section{REFERENCES}

[1] Allen J.F (1988) Maintaining knowledge about temporal intervals. Communications of the ACM 26 (II).

[2] Anahid Bassiri, Ali A. Alesheikh and Mohammad R. Malek (2003) Spatio-Temporal Object modelling in fuzzy Topological Space. PP 1 to 4.

[3] B.C. Chutia and H.C. Chamuah 2015 Application of fuzzy Topological relation in flood predication. IJCA Vol - 122 (USA Ny) PP. 8 to 13.

[4] Dubois and Prade (1980). Fuzzy set and system; Theory and applications Academic Press, New York.
[5] Egenhofer M.J and Franzosa (1991).Point set topological spatial relations. International Journal of Geographic Information System. 5(2): 161-174.

[6] Egenhofer M.J and Herring J.R (1990) A mathematical framework for the definition of topological relationships. In proceedings of the $4^{\text {th }}$ international symposium on Spatial Data Handling K.Brassel and H.Kishimoto(eds), International Geographical Union Zurich PP 803-813.

[7] Lin, Y.M. Luo MK (1997) Fuzzy Topological World Scientific Singapore, 353pp.

[8] Tang Xiniming "Spatial object modelling in fuzzy topological spaces with applications to land cover change" (2004) Jan-China-printed by ITC printing department.

[9] Zadeh L A. Fuzzy sets as a basis for a theory of possibility, Fuzzy Sets and Systems.

[10] Zadeh, L.A, 1965. Fuzzy sets. Information and control 8, 338-353. 